\title{
Remote Vital Sign Recognition through Machine Learning augmented UWB
}

\author{
Soumya Prakash Rana*, Maitreyee Dey*, Robert Brown *, Hafeez Ur Siddiqui*, Sandra Dudley* \\ * School of Engineering, London South Bank University, \\ SE1 0AA London, United Kingdom \\ Email: $\{$ ranas9, deym, dudleyms $\} @ 1$ 1sbu.ac.uk
}

\begin{abstract}
This paper describes an experimental demonstration of machine learning (ML) techniques supplementing radar to distinguish and detect vital signs of users in a domestic environment. This work augments an intelligent location awareness system previously proposed by the authors. That research employed Ultra-Wide Band (UWB) radar complemented by supervised machine learning techniques to remotely identify a persons room location via floor plan training and time stamp correlations. Here, the remote breathing and heartbeat signals are analyzed through Short Term Fourier Transformation (STFT) to determine the Micro-Doppler signature of those vital signs in different room locations. Then, Multi-Class Support Vector Machine (MC-SVM) is implemented to train the system to intelligently distinguish between vital signs during different activities. Statistical analysis of the experimental results supports the proposed algorithm. This work could be used to further understand, for example, how active older people are by engaging in typical domestic activities.

Index Terms-Indoor Positioning System (IPS), Breathing, Heartbeat, Ultra-Wide Band (UWB), Short Term Fourier Transform (STFT), Multi-Class Support Vector Machine (MCSVM)
\end{abstract}

\section{INTRODUCTION}

Indoor Positioning Systems (IPSs) provide a level of intelligence that can be employed for in-building use with IPS finding numerous applications in areas including security, health, assistive living, personalized healthcare etc. IPS locates the position and movement of any objects in an indoor scenario which is often difficult to characterize by non-lineof-sight (N-LOS) of reference objects, presence of obstacles, signal fluctuations or noise, environmental changes, etc. [1]. The proposed work identifies vital signs of persons in different room locations within a domestic setting in an autonomous way. This work analyses and distinguishes the breathing and heartrates of persons and to understand their position in a building under test.

In this paper Ultra-Wide Band (UWB) radar is used to detect users are different locations throughout a home. Recently, many of the localization techniques are based on respiratory motion [2], [3], but few works are based on cardiac-induced radar signatures [4]. Ultra-Wide Band (UWB) radar has advantages over other existing tools due to its non-intrusive, non-tackling capabilities and its potential to penetrate through different materials or obstacles [5] whilst also having very low radio frequency (RF) power levels.

Several studies have investigated UWB-based wireless sensing devices to detect vital signs for health care applications [6]-[9]. Microwave Doppler radar was offered as noninvasive vital signs approximation technique [10]. Though, Doppler radar methods present two difficulties: 1) in penetrat- ing solid and 2) the null point problem citelubecke2002. The most valuable tool in signal analysis is Fourier analysis and it has therefore been used in many fields, though it is limited to static data sequence [11]. The Fourier band describes signals corresponding time-invariant amplitudes and phases over the whole period in which the signal was recorded. An intuitive resolution for dealing with time-dependency is by windowing the signal for specific time instance and calculates its Fourier transform. Then this process repeats for every time stamps. The resulting time-dependent spectrum is known as short time Fourier transformation (STFT) and was first introduced by Gabor [12].

This work proposes and investigates a non-intrusive and intelligent system that can measure and distinguish the vital signs and location of a person in a house under test. UWB radar augmented by machine learning techniques provides a path that a) negates the use of CCTV, thus maintains privacy, b) non-tactile vital sign measurements, thus no wires and c) remotely identifies room usage creating a useful picture of a person's health during the day or night. It has the inbuilt potential to identify changes in behavior and/or vital signs with the help of approved signal processing features. This experiment correlates the position of a person and aims to detect vital signs during normal activities. This proposed method could further assist elderly people get improved into on actual activity levels, etc.

The paper is organized as follows. Section 2 describes the proposed work, including the techniques and algorithms employed for signal analysis machine learning. The experimental set up and result analysis is presented in Section 3. Finally, section 4 draws conclusions on the presented research.

\section{ANALYSIS AND LEARNING}

The proposed work is divided into two stages, signal analysis by STFT and machine learning using MC-SVM. Raw UWB signals are transmitted form a single point in the house under test and return information captured onto a dedicated processing board. The UWB hardware module data can accommodate the multi-path interference, but direct path interferences still occur during data collection process. Thus, gathered signals are pre-processed to eliminate direct path interference between two antennas (transmitter and receiver). The pre-processed signals are subsequently analyzed through STFT, and later the micro-doppler signatures at different time stamps are considered as the employable knowledge for classification using MC-SVM algorithm. These classification results can potentially distinguish who is in a particular room 
and identify possible activity levels by perceiving the changes in vital signs and UWB location methods.

\section{A. Short Term Fourier Transform}

STFT is a powerful, well established and widely used tool for time frequency analysis [12]. In the case here, it used to generate important and distinct time-frequency patterns for respiration and heartbeat rates at different room locations. To determine vital signs in time, the window width must be narrow, alternately the frequency resolution requires longer time spans. The mathematical expression of STFT [13] is shown in Eq. (1) and explained below.

$$
S(a, f)=\sum_{t=-\infty}^{\infty} s(t) u(a-t) e^{-j f n}
$$

Where, $S(a, f)=$ frequency function, $f=$ continuous variable denoting frequency, $u(a-t)=$ shifted frequency, $s(t) u(a-t)=$ short time section of $s(t)$ at time $a$. Here, $s(t)$ is the obtained vital sign signal, which is sampled at $f$ frequency with the duration of $t$. Then, the shifted frequency or window (in our case, this is a hamming window) is convoluted with the short-term section of the signal to observe the frequency changes within a short term.

\section{B. Multi-Class Support Vector Machine}

The UWB vital sign data produced from the indoor scenario here is considered a multi-class categorization case. Therefore the extracted features are labelled and fed into a Crammer and Singer's MC-SVM, where a set of labelled training pattern is represented by $\left(x_{1}, y_{1}\right), \ldots,\left(x_{l}, y_{l}\right)$ of cardinality $l$, where $x_{i} \in R^{d}$ and $y_{i} \in 1, \ldots, k, w \in R^{d}$ is the weight vector, $C \in R_{+}$is the regularization constant, and $\varphi$ is mapping function which projects training pattern into a suitable feature space $H$ that allows for nonlinear decision surfaces. Crammer and Singer [14] proposed a SVM with multi categorization ability by solving the quadratic optimization problem,

$$
\begin{aligned}
& \min _{w_{m} \in H, \xi \in R^{l}} \frac{1}{2} \sum_{m=1}^{k} w_{m}^{T} w_{m}+C \sum_{i=1}^{l} \xi_{i} \\
& \text { subject to } \quad w_{y_{i}}^{T} \varphi\left(x_{i}\right)-w_{t}^{T} \varphi\left(x_{i}\right) \geq 1-\delta_{y_{i}, t}-\xi_{i} \\
& \quad i=1, \ldots, l, t \in 1, \ldots, k
\end{aligned}
$$

where, $\delta_{i, j}, j$ is the Kronecker delta, defined as 1 for $i=j$ and as 0 otherwise. The resultant decision function is defined as,

$$
\operatorname{argmax}_{m} f_{m}(x)=\operatorname{argmax}_{m} w_{m}^{T} \varphi(x)
$$

Note that the constraints $\xi_{i} \geq 0, i=1, \ldots, l$, are implicitly indicated in the margin constraints of (2) when $t$ equals $y_{i}$. In addition, (2) focuses on classification rule (3) without any bias terms. A non-zero bias term can be easily modelled by including an additional constant feature to each $x$. Therefore, different categories of data are classified by solving this decision function and the results are analysed in the following section.

\section{EXPERIMENT \& RESULT ANALYSIS}

The UWB vital sign monitoring experiment has been carried out on the ground floor area of a semi-detached house located in Essex, UK. The ground floor plan is shown in Fig. 1 and comprises four rooms: a living room, kitchen, dining room, and a downstairs bathroom. The single UWB device is fixed towards the back corner of the living room (top left area in Fig. 1).

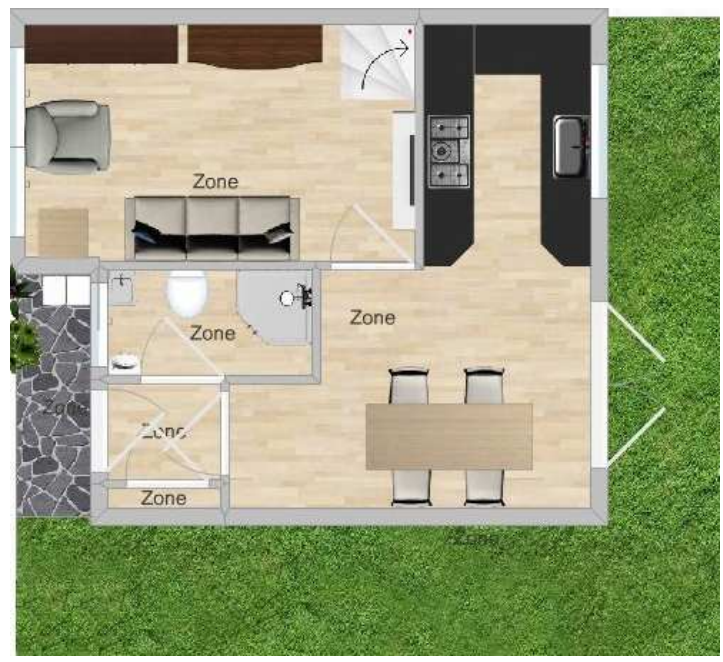

Fig. 1. The floor plan where UWB experiment was executed

A Time Domain PulsON 410 (P410) (shown in Fig. 2) is the UWB hardware module employed for data acquisition, which is short-range radar with $1.4 \mathrm{GHz}$ of RF bandwidth. This P410 commercial radar module, embedded with inhouse developed software was connected to a raspberry pi which stored the time stamped radar data [15]. The experiment was carried out in the house described earlier and compared to diary measurements made at the time to correlate findings. The transceiver transmits RF from 3.1 $\mathrm{GHz}$ to $5.3 \mathrm{GHz}$, with its center frequency at $4.3 \mathrm{GHz}$, and follows Federal Communications Commission (FCC) restrictions [16].

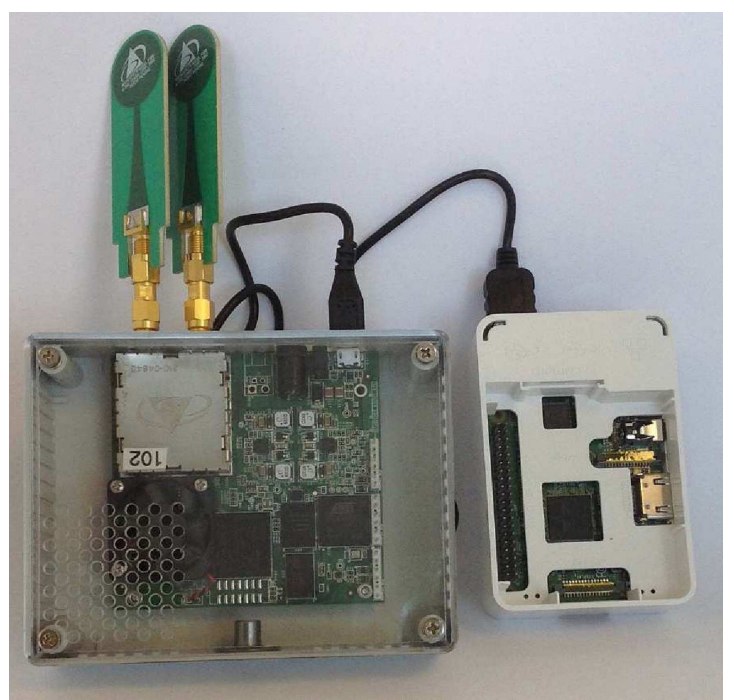

Fig. 2. The P410 hardware module used in the experiment 
The experiment was carried out by Matlab R2016b tool in a Intel ${ }^{R}$ Core $^{T M}$ i7 processor@ $3.60 \mathrm{GHz}$ based Windows 7 Enterprise 64 bit operating system and it has $7856 \mathrm{MB}$ NVIDIA Graphics Processing Unit (GPU).

This work build on that presented in [17], where UWB radar signals are learned though supervised machine learning algorithms to remotely determine and pivotally recognize the room position information of persons at different times of a day. Therefore, the MC-SVM is already trained with the time stamp and localization information required here. This experiment investigates the use of machine learning augmented UWB to detect and recognize the vital sign patterns of a person in different rooms during those time stamps. Based on this prior knowledge it is indeed found that machine learning when appropriately applied assists in the distinction of vital sign patterns in different room locations. The parameters and configuration used for this experiment are tabulated in Table I.

TABLE I

PARAMETER SETTING FOR THE EXPERIMENT

\begin{tabular}{|ll|}
\hline Radar \& Lab Based & Values \\
\hline Radar range & 10 Meters \\
Antenna mode & $1 T_{x}$ and $1 R_{x}$ \\
Transmission gain & $63 \mathrm{~dB}$ \\
Scan time interval & 0.012 Seconds \\
Sampling rate & $0.3063 \mathrm{MHz}$ \\
Resolution of each scan & 1440 \\
Total no of scans & 12222 \\
Window type & Hamming \\
\hline
\end{tabular}

The raw data from the four different rooms mentioned were accumulated over different time stamps via the set-up described. The data is accumulated with the presence and absence of persons where the rest of the environment is assumed static. Therefore, eight distinct breathing and heartbeats are found during the data collection. The description of different vital signs is included in Table II.

TABLE II

DIFFERENT PATTERNS AND THEIR DESCRIPTIONS FOR THE EXPERIMENT

\begin{tabular}{|ll|}
\hline Position Details & Vital Sign Patterns \\
\hline Living room & Respiration \\
Living room & Heartbeat \\
Kitchen & Respiration \\
Kitchen & Heartbeat \\
Dinning & Respiration \\
Dinning & Heartbeat \\
Bathroom & Respiration \\
Bathroom & Heartbeat \\
\hline
\end{tabular}

Amplitude fluctuations are captured when persons or moving objects are in the LOS of signal propagation from the radar. Time frequency analysis is executed using STFT to extract human breathing and heartbeat rates. As the general rates of these vital signs are already widely known, thresholds are fixed to distinguish between them. Later these microdoppler signatures are learned by MC-SVM, enabling vital sign distinction between persons and knowledge of their location information along with pivotal time information. Fig. 3 , shows the example of a time frequency variation of a respiration signal during the moving window analysis.

Fig. 3 shows the windowing frequency information of a signal. The incoming raw data signal is divided into six parts

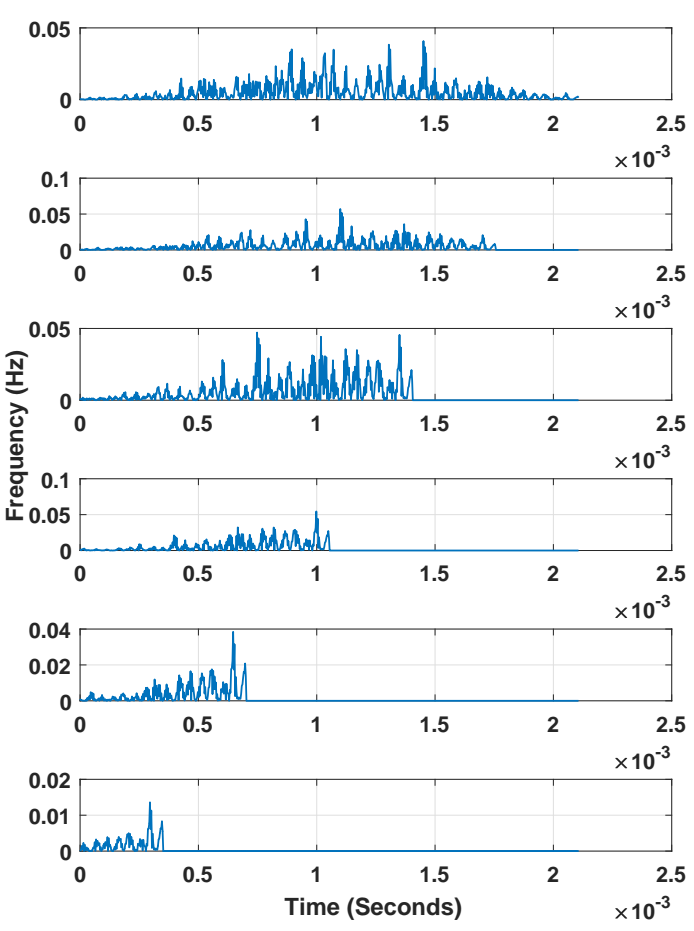

Fig. 3. Moving window analysis of a breathing signal

and a hamming window is used for convolution. It is clearly seen that the frequencies repeat themselves in subsequent windows. After doing the final summation of discrete time STFT, it produces a breathing pattern that also occurs as the person is sitting watching television. The same UWB data (and TV sensor) is being used for location identification and vital sign determination. Fig. 4 shows the breathing pattern for that person in resting mode in this case.The maximum peak occurs around $0.24 \mathrm{~Hz}$, which signifies a breathing rate for this person of 14.4 per minute.

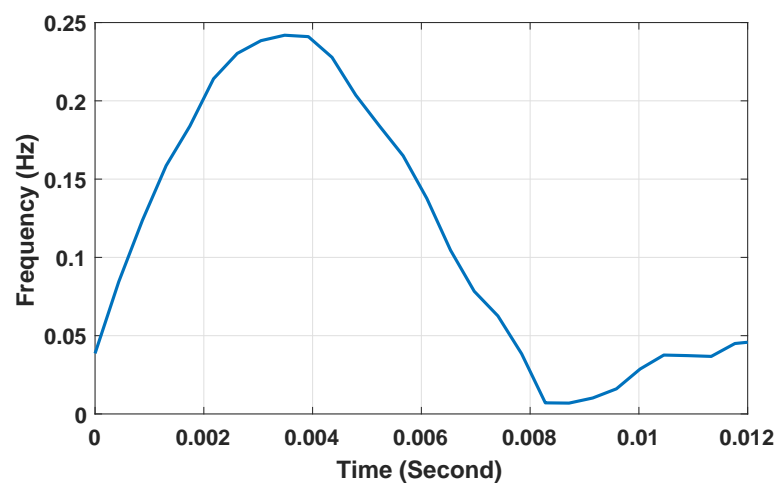

Fig. 4. UWB breathing pattern for the person in resting mode

Fig. 5, shows the frequency analysis of a heartbeat signal for the same person through moving window during the STFT. Each segment of the signal has the interval of 2.5 millisecond and the frequencies occurred within the range 
of 0 to $0.1 \mathrm{~Hz}$. After STFT the micro-doppler signature of a heartbeat signal is found for the experimented person in living room, and is shown in Fig. 6.

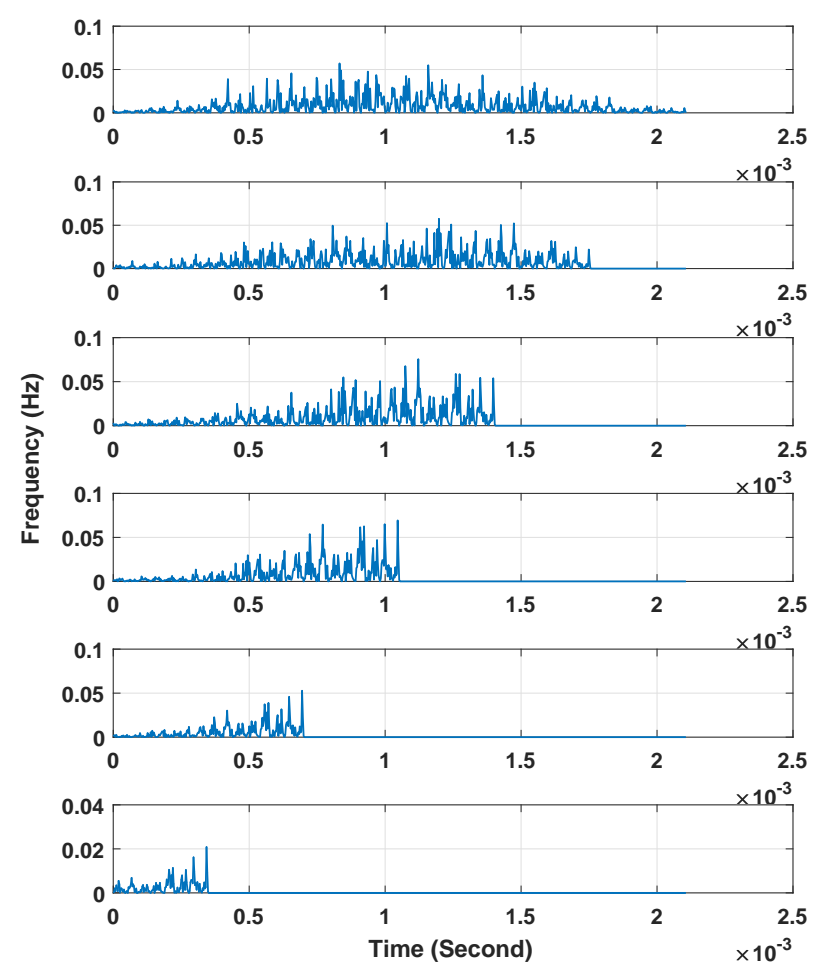

Fig. 5. Moving window analysis of a heartbeat signal

Each heartbeat signal has the duration of 0.012 seconds and the peak is found around in $1.3 \mathrm{~Hz}$. It indicates the person has the heartbeat rate of 78 beats per minute. The rate of the respiration and heartbeat of each person is verified by a medical measurement mobile App. The experimental results compared and support the actual rate obtained from the App.

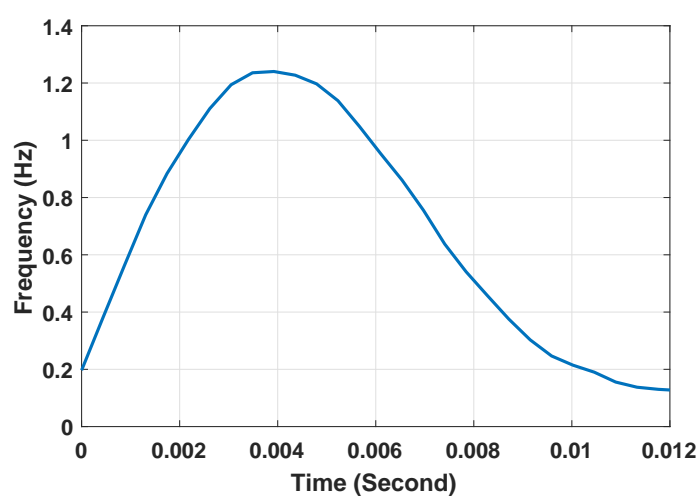

Fig. 6. UWB heartbeat pattern for the person in resting mode

In the second experimental phase, these vital signs are learned through the supervised machine learning method MC-SVM. This employed to distinguish persons via the micro-doppler signature of their breathing and heartbeat.
Table III, shows the classification results of different classes described in Table II.

TABLE III

ClassificAtion BREATHING AND HeARTbeat Signals Results by MC-SVM FOR DIFFERENT ROOMS

\begin{tabular}{|llll|}
\hline Statistical Measurements & $10 \%$ & $20 \%$ & $30 \%$ \\
\hline Correct Rate & 0.9827 & $\mathbf{0 . 9 8 3 0}$ & 0.9829 \\
Error Rate & 0.0173 & 0.0170 & 0.0171 \\
Sensitivity & 1.0000 & 1.0000 & 1.0000 \\
Specificity & 1.0000 & 1.0000 & 1.0000 \\
Positive Predictive Value & 1.0000 & 1.0000 & 1.0000 \\
Negative Predictive Value & 1.0000 & 1.0000 & 1.0000 \\
Time elapsed (in Seconds) & 0.7605 & 0.7604 & 0.7602 \\
\hline
\end{tabular}

Table III shows that the proposed vital sign prediction model provided the highest testing correction rate 0.9830 (marked in bold) and lowest error rate 0.0170 in the $20 \%$ percent training data. Testing correction rate was increased from 0.9827 to 0.9830 for $10 \%$ to $20 \%$ training data. In case of $30 \%$ training data, the algorithm is being over-fitted due to the high dimensional data points, and testing accuracy falls to 0.9829 and the error rate increases to 0.0171 . The objective of the proposed method is to fit the model, so that it could make valid predictions on untrained or test data. Therefore, the performance of the proposed algorithm at $20 \%$ training data is considered as the performance of the model.

Other evaluation parameters are also determined to support the robustness of the model. In this case (20\% training and $80 \%$ testing data), sensitivity 1.0000 of the proposed algorithm indicates the probability of correctly identifying the person through their vital signs. Additionally, specificity of 1.0000 tells the probability of the system to recognize the person accurately when there is no known person occurs. The Positive Predictive Value (PPV) 1.0000 signifies the probability that the system gives positive result about the person's identity which is true. Negative Predictive Value (NPV) 1.0000 points out the probability that system gives negative result about the person's identity and it is true.

Thus, the experimental results achieved $98.3 \%$ accuracy for distinguishing a person's room location through their vital signs with a small training set. This method can potentially now be improved to recognize, whether the person is doing some activity or resting by the positive and negative classification results. Also the accuracy over the attempt is made by the system traced by their result analysis.

\section{CONClusion AND Future Work}

Breathing and heartbeat signatures are two important vital signs of a human body which are estimated in this work with the non-intrusive UWB radar and the application of a machine learning algorithms that could alongside other sensors such as smart plugs can potentially recognize the person's activity type and correlated with location information therefore, the proposed method can be used to observe and track elderly people for example, with tailored feedback and suggestions on their daily personal activity levels via mobile devices. That can inform, empower and encourage older adults to remain active to improve their well-being, and provide help when they need. Further work is underway to extend this study with multiple occupants and longer time intervals. Along with this, distinguishing occupant and 
activity patterns aligned with vital sign measurements will be explored in the currently tested environments to provide real activities and effective measurement through non-tactile, non-tag wearing methods.

\section{ACKNOWLEDGMENT}

The work carried out here is part of the Digital Agent Networking for Customer Energy Reduction (DANCER) project, funded by the UK EPSRC Energy Efficiency in Buildings programme (EP/K002473/1).

\section{REFERENCES}

[1] M. Švecová and D. Kocur, "Taylor series-based tracking algorithm for through wall tracking of a moving persons," Acta Polytechnica Hungarica, vol. 7, no. 1, pp. 5-21, 2010.

[2] A. Nezirovic, A. G. Yarovoy, and L. P. Ligthart, "Signal processing for improved detection of trapped victims using uwb radar," IEEE Transactions on Geoscience and Remote Sensing, vol. 48, no. 4, pp. 2005-2014, 2010.

[3] J. Li, Z. Zeng, J. Sun, and F. Liu, "Through-wall detection of human being's movement by uwb radar," IEEE Geoscience and Remote Sensing Letters, vol. 9, no. 6, pp. 1079-1083, 2012.

[4] F. Thiel, M. Hein, U. Schwarz, J. Sachs, T. Lindel, and F. Seifert, "Exploring the benefits of ultra-wideband radar for high-and ultra-high field magnetic resonance imaging," in Microwave Conference, 2009. EuMC 2009. European. IEEE, 2009, pp. 866-869.

[5] P. Withington, H. Fluhler, and S. Nag, "Enhancing homeland security with advanced uwb sensors," IEEE Microwave magazine, vol. 4, no. 3, pp. 51-58, 2003.

[6] E. M. Staderini, "Uwb radars in medicine," IEEE Aerospace and Electronic Systems Magazine, vol. 17, no. 1, pp. 13-18, 2002.

[7] C. G. Bilich, "Bio-medical sensing using ultra wideband communications and radar technology: A feasibility study," in Pervasive Health Conference and Workshops, 2006. IEEE, 2006, pp. 1-9.

[8] G. Ossberger, T. Buchegger, E. Schimback, A. Stelzer, and R. Weigel, "Non-invasive respiratory movement detection and monitoring of hidden humans using ultra wideband pulse radar," in Ultra Wideband Systems, 2004. Joint with Conference on Ultrawideband Systems and Technologies. Joint UWBST \& IWUWBS. 2004 International Workshop on. IEEE, 2004, pp. 395-399.

[9] S. Venkatesh, C. R. Anderson, N. V. Rivera, and R. M. Buehrer "Implementation and analysis of respiration-rate estimation using impulse-based uwb," in Military Communications Conference, 2005. MILCOM 2005. IEEE. IEEE, 2005, pp. 3314-3320.

[10] S. Ivashov, V. Razevig, A. Sheyko, and I. Vasilyev, "Detection of human breathing and heartbeat by remote radar," in Progress in Electromagnetic Research Symposium, vol. 2004, 2004.

[11] E. C. Titchmarsh et al., Introduction to the theory of Fourier integrals. Clarendon Press Oxford, 1948, vol. 2.

[12] D. Gabor, "Theory of communication," Journal of Institution of Electrical Engineers, vol. 93, no. 3, pp. 429-457, 1946.

[13] S. H. Nawab and T. F. Quatieri, "Short-time fourier transform," in Advanced topics in signal processing. Prentice-Hall, Inc., 1987, pp. 289-337.

[14] K. Crammer and Y. Singer, "On the algorithmic implementation of multiclass kernel-based vector machines," Journal of machine learning research, vol. 2, no. Dec, pp. 265-292, 2001.

[15] R. Brown, N. Ghavami, M. Adjrad, M. Ghavami, S. Dudley et al., "Occupancy based household energy disaggregation using ultra wideband radar and electrical signature profiles," Energy and Buildings, vol. 141, pp. 134-141, 2017.

[16] F. C. Commission, "In the matter of revision of part 15 of the commission's rules regarding ultra-wideband transmission systems," First Report And Order, ET Docket 98-153, 2002.

[17] S. P. Rana, M. Dey, H. Siddiqui, G. Tiberi, M. Ghavami, and S. Dudley, "UWB localization employing supervised learning method," in IEEE International Conference on Ubiquitous Wireless Broadband ICUWB2017, Salamanca, Spain, September 2017. 